IJMMS 28:10 (2001) 571-580

PII. S0161171201007153

http://ijmms.hindawi.com

(C) Hindawi Publishing Corp.

\title{
MOTION OF TWO POINT VORTICES IN A STEADY, LINEAR, AND ELLIPTICAL FLOW
}

\author{
ROBERT M. GETHNER
}

(Received 19 March 2001)

\begin{abstract}
For a pair of point vortices in an inviscid, incompressible fluid in the plane, the relative and absolute motion are determined when the vortices move under the influence of (1) each other, and (2) a steady, linear, and elliptical background flow.
\end{abstract}

2001 Mathematics Subject Classification. 76B47.

1. Introduction. The point vortex model $[1,4,5]$ is an idealization of the motion of a collection of vortices in an inviscid, incompressible fluid in the plane. Each vortex is assumed to be a point, and to induce in the surrounding fluid a velocity field, namely that of a Rankine vortex whose core has shrunk to a point. Each such point $P$ moves with a velocity equal to the sum of the velocities induced by the other points, and the velocity field induced by $P$ moves, without change of form, with the same velocity as $P$ itself.

We investigate the absolute and relative motion in the plane of a pair of point vortices that are embedded in a steady flow whose velocity field has the form

$$
\langle-\alpha y, \beta x\rangle,
$$

where $\alpha$ and $\beta$ are constants such that $\alpha \geq \beta>0$. The flow (1.1) carries fluid particles counterclockwise around the origin, in elliptical trajectories. Kimura and Hasimoto [3] have analyzed a similar problem in which two vortices move in a simple shear flow $\langle\alpha y, 0\rangle$. They require their vortices to be identical; here that requirement is dropped.

Here are the basic equations and notation needed for our analysis.

First, we need some information about the flow (1.1) (henceforth called the "background flow"). The position $\langle x, y\rangle$ of a given fluid particle in the background flow satisfies the equations

$$
\frac{d x}{d t}=-\alpha y, \quad \frac{d y}{d t}=\beta x,
$$

which have a general solution

$$
x=x_{0} \cos \omega t-D y_{0} \sin \omega t, \quad y=D^{-1} x_{0} \sin \omega t+y_{0} \cos \omega t,
$$

where $x_{0}=x(0), y_{0}=y(0)$, and

$$
D=\sqrt{\frac{\alpha}{\beta}}, \quad \omega=\sqrt{\alpha \beta} .
$$


Thus, a fluid particle that begins at $\left(x_{0}, y_{0}\right)$ will complete one counterclockwise revolution around the ellipse $x^{2} / \alpha+y^{2} / \beta=x_{0}^{2} / \alpha+y_{0}^{2} / \beta$ in time $2 \pi / \sqrt{\alpha \beta}$.

It follows from (1.3) that the linear transformation $L_{t}: \mathbb{R}^{2} \rightarrow \mathbb{R}^{2}$, defined by

$$
L_{t}\left(\left[\begin{array}{l}
X \\
Y
\end{array}\right]\right)=\left[\begin{array}{cc}
\cos \omega t & -D \sin \omega t \\
D^{-1} \sin \omega t & \cos \omega t
\end{array}\right]\left[\begin{array}{l}
X \\
Y
\end{array}\right],
$$

takes as input the location of a given fluid particle in the background flow at time 0 , and gives as output the particle's location at time $t$. The inverse transformation

$$
L_{t}^{-1}\left(\left[\begin{array}{l}
x \\
y
\end{array}\right]\right)=\left[\begin{array}{cc}
\cos \omega t & D \sin \omega t \\
-D^{-1} \sin \omega t & \cos \omega t
\end{array}\right]\left[\begin{array}{l}
x \\
y
\end{array}\right]
$$

takes as input the location of a given fluid particle in the background flow at time $t$, and gives as output the particle's location at time 0 .

Next, we introduce the equations of motion of the vortices. Denote by $\left\langle x_{j}, y_{j}\right\rangle$ $(j=1,2)$ the position of the $j$ th vortex, and put

$$
r=\sqrt{\left(x_{2}-x_{1}\right)^{2}+\left(y_{2}-y_{1}\right)^{2}} .
$$

Then, because the velocity of each vortex is the sum of the background flow's velocity and the velocity induced by the other vortex, the vortices' positions satisfy the following differential equations:

$$
\begin{aligned}
& \frac{d x_{1}}{d t}=\kappa_{2} \frac{y_{2}-y_{1}}{r^{2}}-\alpha y_{1} ; \\
& \frac{d y_{1}}{d t}=-\kappa_{2} \frac{x_{2}-x_{1}}{r^{2}}+\beta x_{1} ; \\
& \frac{d x_{2}}{d t}=-\kappa_{1} \frac{y_{2}-y_{1}}{r^{2}}-\alpha y_{2} ; \\
& \frac{d y_{2}}{d t}=\kappa_{1} \frac{x_{2}-x_{1}}{r^{2}}+\beta x_{2} ;
\end{aligned}
$$

here $\kappa_{1}$ and $\kappa_{2}$ are nonzero constants.

Finally, to obtain differential equations for the vortices' relative position, we first define

$$
\xi=x_{2}-x_{1}, \quad \eta=y_{2}-y_{1}, \quad \kappa=\kappa_{1}+\kappa_{2}
$$

then, by subtracting (1.8) from (1.10) and (1.9) from (1.11), we get

$$
\begin{aligned}
& \frac{d \xi}{d t}=-\left(\frac{\kappa}{r^{2}}+\alpha\right) \eta, \\
& \frac{d \eta}{d t}=\left(\frac{\kappa}{r^{2}}+\beta\right) \xi .
\end{aligned}
$$

The system (1.13) has a Hamiltonian

$$
H=-\frac{1}{2}\left[\kappa \log \left(\xi^{2}+\eta^{2}\right)+\beta \xi^{2}+\alpha \eta^{2}\right]
$$

that is, $\partial H / \partial \eta$ equals the right-hand side of (1.13a) and $-\partial H / \partial \xi$ equals the right-hand side of (1.13b). Each solution curve of (1.13) is contained in a level curve of $H$. (Cf. [6, pages 43-45] for an introduction to Hamiltonians.) 
In polar coordinates $r$ and $\theta$ defined by

$$
\xi=r \cos \theta, \quad \eta=r \sin \theta
$$

where $r$ satisfies (1.7), equations (1.13) and (1.14) take the form

$$
\begin{gathered}
\frac{d r}{d t}=-2^{-1}(\alpha-\beta) r \sin 2 \theta, \\
\frac{d \theta}{d t}=\frac{\kappa}{r^{2}}+\alpha \sin ^{2} \theta+\beta \cos ^{2} \theta \\
H=-2^{-1}\left[2 \kappa \log r+\beta r^{2} \cos ^{2} \theta+\alpha r^{2} \sin ^{2} \theta\right] .
\end{gathered}
$$

We are now ready to begin our analysis. In Section 2, we consider absolute motion; we consider relative motion in Sections 3.1, 3.2, 3.3, and 3.4. The character of the relative motion depends on whether $\alpha=\beta$ (when the background flow is solid-body rotation) or $\alpha>\beta$ (when the background flow is elliptical but not circular); in the latter case the behavior depends on the sign of $\kappa$.

2. Absolute motion. Theorems 2.1 and 2.2 below describe the absolute motion in the cases $\kappa \neq 0$ and $\kappa=0$, respectively.

For $\kappa \neq 0$, the center of vorticity of the vortices $\left\langle x_{j}, y_{j}\right\rangle(j=1,2)$ is defined to be $\left\langle x_{c}, y_{c}\right\rangle$, where

$$
x_{c}=\kappa^{-1}\left(\kappa_{1} x_{1}+\kappa_{2} x_{2}\right), \quad y_{c}=\kappa^{-1}\left(\kappa_{1} y_{1}+\kappa_{2} y_{2}\right)
$$

THEOREM 2.1. Fix $\alpha$ and $\beta$, where $\alpha \geq \beta>0$, and let $\left\langle x_{j}, y_{j}\right\rangle(j=1,2)$ be a solution of the system (1.8), (1.9), (1.10), and (1.11). If $\kappa$ defined by (1.12) is nonzero, then the center of vorticity moves with the background flow.

Proof. By computing $\kappa^{-1}\left\{\kappa_{1}[(1.8)]+\kappa_{2}[(1.10)]\right\}$, we find that $d x_{c} / d t=-\alpha y_{c}$. Similarly, $d y_{c} / d t=\beta y_{c}$. Thus, since the background flow is given by (1.1), the proof is complete.

THEOREM 2.2. Fix $\alpha$ and $\beta$, with $\alpha \geq \beta>0$, and pick real numbers $\kappa_{1}$ and $\kappa_{2}$ such that $\kappa=\kappa_{1}+\kappa_{2}=0$. Define $D, \omega$, and $L_{t}$ by (1.4) and (1.5). Finally, choose real numbers $X_{1}, Y_{1}, X_{2}$, and $Y_{2}$, with $\left(X_{2}-X_{1}\right)^{2}+\left(Y_{2}-Y_{1}\right)^{2} \neq 0$, and set $\xi_{0}=X_{2}-X_{1}$ and $\eta_{0}=Y_{2}-Y_{1}$.

Then the system (1.7), (1.8), (1.9), (1.10), and (1.11) has a unique solution satisfying $x_{j}(0)=X_{j}$ and $y_{j}(0)=Y_{j}(j=1,2)$; that solution is

$$
\left[\begin{array}{l}
x_{j} \\
y_{j}
\end{array}\right]=L_{t}\left(\left[\begin{array}{c}
X_{j} \\
Y_{j}
\end{array}\right]\right)+\frac{\kappa_{1}}{D^{-1} \xi_{0}^{2}+D \eta_{0}^{2}}\left\{t L_{t}\left(\left[\begin{array}{c}
-D \eta_{0} \\
D^{-1} \xi_{0}
\end{array}\right]\right)+G(t) L_{t}\left(\left[\begin{array}{l}
\xi_{0} \\
\eta_{0}
\end{array}\right]\right)\right\},
$$

where

$$
\begin{aligned}
G(t)=-\frac{D-D^{-1}}{2(\alpha-\beta)} \log \left\{\left(\xi_{0}^{2}+\eta_{0}^{2}\right)^{-1} \cdot[\right. & \left(\xi_{0}^{2}+\eta_{0}^{2}\right) \cos ^{2} \omega t-\left(D-D^{-1}\right) \xi_{0} \eta_{0} \sin 2 \omega t \\
+ & \left.\left.\left(D^{-2} \xi_{0}^{2}+D^{2} \eta_{0}^{2}\right) \sin ^{2} \omega t\right]\right\} .
\end{aligned}
$$


Proof. We will rewrite the system (1.8), (1.9), (1.10), and (1.11) in terms of new variables $\hat{x}_{j}$ and $\hat{y}_{j}$ defined by

$$
\left[\begin{array}{l}
\hat{x}_{j} \\
\hat{y}_{j}
\end{array}\right]=L_{t}^{-1}\left(\left[\begin{array}{l}
x_{j} \\
y_{j}
\end{array}\right]\right) .
$$

We hope in this way to simplify the system by eliminating (or at least reducing) the effect of the background flow.

To convert (1.8), (1.9), (1.10), and (1.11) to the new variables, we first rewrite (2.4) as

$$
\hat{x}_{j}=x_{j} \cos \omega t+D y_{j} \sin \omega t, \quad \hat{y}_{j}=-D^{-1} x_{j} \sin \omega t+y_{j} \cos \omega t .
$$

We then differentiate the four equations in (2.5) with respect to $t$, use (1.8), (1.9), (1.10), and (1.11) to eliminate the derivatives of $x_{j}$ and $y_{j}$, and apply (1.12), (1.4), and the condition $\kappa=0$; the result is

$$
\frac{d \hat{x}_{j}}{d t}=\kappa_{1} \frac{(-\eta \cos \omega t+D \xi \sin \omega t)}{r^{2}}, \quad \frac{d \hat{y}_{j}}{d t}=\kappa_{1} \frac{\left(D^{-1} \eta \sin \omega t+\xi \cos \omega t\right)}{r^{2}} .
$$

Now by (1.13),

$$
\frac{d \xi}{d t}=-\alpha \eta, \quad \frac{d \eta}{d t}=\beta \xi
$$

This last system is just (1.2) with $x$ and $y$ replaced by $\xi$ and $\eta$; thus, by (1.3), the definitions of $\xi_{0}$ and $\eta_{0}$, and (1.12), the general solution of (2.7) is

$$
\xi=\xi_{0} \cos \omega t-D \eta_{0} \sin \omega t, \quad \eta=D^{-1} \xi_{0} \sin \omega t+\eta_{0} \cos \omega t .
$$

After solving (2.8) for $\cos \omega t$ and $\sin \omega t$ and substituting the result into (2.6), we obtain

$$
\begin{aligned}
& \frac{d \hat{x}_{j}}{d t}=\kappa_{1}\left(D^{-1} \xi_{0}^{2}+D \eta_{0}^{2}\right)^{-1}\left[-D \eta_{0}+\left(D-D^{-1}\right) \xi_{0} \frac{\xi \eta}{\left(\xi^{2}+\eta^{2}\right)}\right], \\
& \frac{d \hat{y}_{j}}{d t}=\kappa_{1}\left(D^{-1} \xi_{0}^{2}+D \eta_{0}^{2}\right)^{-1}\left[D^{-1} \xi_{0}+\left(D-D^{-1}\right) \eta_{0} \frac{\xi \eta}{\left(\xi^{2}+\eta^{2}\right)}\right] .
\end{aligned}
$$

But by (2.7), $(d / d t)\left(\xi^{2}+\eta^{2}\right)=-2(\alpha-\beta) \xi \eta$. This last equation allows us to integrate (2.9), after which, using (2.3) and (2.8), we find that

$$
\begin{aligned}
& \hat{x}_{j}=\hat{x}_{j}(0)+\kappa_{1}\left(D^{-1} \xi_{0}^{2}+D \eta_{0}^{2}\right)^{-1}\left[-D \eta_{0} t+\xi_{0} G(t)\right], \\
& \hat{y}_{j}=\hat{y}_{j}(0)+\kappa_{1}\left(D^{-1} \xi_{0}^{2}+D \eta_{0}^{2}\right)^{-1}\left[D^{-1} \xi_{0} t+\eta_{0} G(t)\right] .
\end{aligned}
$$

Finally, we put (2.10) into matrix form and apply $L_{t}$ to both sides; (2.2) then follows because, by (2.5), $\hat{x}_{j}(0)=x_{j}(0)$ and $\hat{y}_{j}(0)=y_{j}(0)$. This completes the proof of Theorem 2.2.

COROLLARY 2.3. Under the hypotheses of Theorem 2.2,

$$
\left[\begin{array}{l}
x_{j} \\
y_{j}
\end{array}\right]=\frac{\kappa_{1} t}{D^{-1} \xi_{0}^{2}+D \eta_{0}^{2}} L_{t}\left(\left[\begin{array}{c}
-D \eta_{0} \\
D^{-1} \xi_{0}
\end{array}\right]\right)+O(1) .
$$


Proof. This follows trivially from (2.2), (2.3), and (2.5).

From Corollary 2.3, along with (1.4) and the interpretation of $L_{t}$ given in Section 1, it follows that, when $\kappa=0$, the two vortices move in a spiral around and away from the origin. More precisely, each vortex stays a bounded distance from a moving point which behaves as follows:

(a) it moves counterclockwise around the origin with period $2 \pi / \sqrt{\alpha \beta}$;

(b) it lies, at time $t$, on the ellipse

$$
\frac{x^{2}}{\alpha}+\frac{y^{2}}{\beta}=\left(\kappa_{1} t\right)^{2} \frac{\left(D^{2} \eta_{0}^{2} / \alpha+D^{-2} \xi_{0}^{2} / \beta\right)}{\left(D^{-1} \xi_{0}^{2}+D \eta_{0}^{2}\right)^{2}} .
$$

\section{Relative motion}

3.1. The case $\alpha=\beta$. The following theorem is a direct consequence of (1.16).

THEOREM 3.1. Fix real numbers $\alpha, \beta$, and $\kappa$ such that $\alpha=\beta>0$, and consider a pair of vortices whose positions satisfy equations (1.7), (1.8), (1.9), (1.10), and (1.11). The line segment joining the two vortices has constant length and rotates with constant, possibly zero, angular velocity $\kappa r_{0}^{-2}+\alpha$, where $r_{0}$ is the segment's length.

3.2. The case $\kappa=0$. From the proof of Theorem 2.2 (see (2.7) and (2.8)) we have the following result.

Theorem 3.2. Fix real numbers $\alpha, \beta, \kappa_{1}$, and $\kappa_{2}$ such that $\alpha \geq \beta>0$ and $\kappa=$ $\kappa_{1}+\kappa_{2}=0$, and consider a pair of vortices whose positions satisfy equations (1.7), (1.8), (1.9), (1.10), and (1.11). In ( $\xi, \eta)$-coordinates (1.12), the second vortex moves around the first, with period $2 \pi / \sqrt{\alpha \beta}$, on the ellipse $\xi^{2} / \alpha+\eta^{2} / \beta=\xi_{0}^{2} / \alpha+\eta_{0}^{2} / \beta$.

Theorems 3.1 and 3.2 agree in the case where $\alpha=\beta$ and $\kappa=0$.

3.3. The case $\alpha>\beta, \kappa>0$. Our investigation of the motion when $\alpha>\beta$ and $\kappa \neq 0$ depends on understanding the level curves of the Hamiltonian $H$ in (1.17), which in turn requires us to analyze the function

$$
g_{w}(r)=(\alpha-\beta)^{-1}\left[4 r^{-2}(\kappa \log r+w)+\alpha+\beta\right] .
$$

For $\kappa>0$, the following lemma gives the information we need.

LEMMA 3.3. Pick $\alpha, \beta$, and $\kappa$, with $\alpha>\beta>0$ and $\kappa>0$, and define $g_{w}(r)$ by (3.1). Set

$$
r^{*}=r^{*}(w)=e^{1 / 2-w / \kappa} .
$$

Then,

(a) $g_{w}$ is increasing on $\left(0, r^{*}\right]$; $\lim _{r \rightarrow 0^{+}} g_{w}(r)=-\infty ; g_{w}(r)>1$ for $r \geq r^{*}$;

(b) given a number $u$ in $[-1,1]$, the equation $g_{w}(r)=u$ has exactly one solution $r$ in $(0, \infty]$, namely, $r=f_{w}(u)$, where $f_{w}$ is the inverse function of the restriction of $g_{w}(r)$ to the interval $\left(0, r^{*}\right]$;

(c) $0<f_{w}(u) \leq r^{*}$ for all real $w$ and all $u$ in $[-1,1]$;

(d) for each real $w$, and each fixed $u$ in $[-1,1], f_{w}(u)$ is a decreasing function of $w$;

(e) $\lim _{w \rightarrow \infty} f_{w}(u)=0$ and $\lim _{w \rightarrow-\infty} f_{w}(u)=+\infty$, uniformly for $u$ in $[-1,1]$. 
Proof. The first two statements in (a) are obvious; the third holds because (i) $g_{w}$ is decreasing for $r \geq r^{*}$, while (ii) $\lim _{r \rightarrow \infty} g_{w}(r)>1$. Part (b) follows immediately from (a), and (c) from (b).

To prove (d), we fix $u$ in $[-1,1]$ and pick real numbers $z$ and $w$ such that $z<w$. Then $f_{z}(u)>f_{w}(u)$; otherwise, since $g_{w}(r)$ is an increasing function of $r \leq r^{*}$ for fixed $w$, and an increasing function of $w$ for fixed $r$, we would have

$$
u=g_{z}\left(f_{z}(u)\right) \leq g_{z}\left(f_{w}(u)\right)<g_{w}\left(f_{w}(u)\right)=u .
$$

The first limit in (e) is a consequence of (c) and (3.2). To establish the second limit, we first calculate, using (3.1), that $g_{w}(\sqrt[3]{-w}) \rightarrow-\infty$ as $w \rightarrow-\infty$; thus, when $w$ is a sufficiently large negative, $u>g_{w}(\sqrt[3]{-w})$ for all $u$ in $[-1,1]$. The second limit then follows when we apply $f_{w}$ to this last inequality. This completes the proof of (e) and of Lemma 3.3.

THEOREM 3.4. Fix real numbers $\alpha, \beta, \kappa_{1}$, and $\kappa_{2}$ such that $\alpha>\beta>0$ and $\kappa=\kappa_{1}+\kappa_{2}>$ 0 , and consider a pair of vortices whose positions satisfy (1.7), (1.8), (1.9), (1.10), and (1.11). In $(\xi, \eta)$-coordinates, the second vortex moves around the first counterclockwise in a simple closed curve, with period

$$
T=8 \int_{0}^{\pi / 2} \frac{d \theta}{2 \kappa /\left[f_{w}(\cos 2 \theta)\right]^{2}+(\alpha+\beta)-(\alpha-\beta) \cos 2 \theta} .
$$

The period $T$ is a decreasing function of $w$ such that $T \rightarrow 2 \pi / \sqrt{\alpha \beta}$ as $w \rightarrow-\infty$, and $T \rightarrow 0$ as $w \rightarrow \infty$. The maximum separation $r$ of the vortices occurs when $\theta=0, \pi$, and the minimum when $\theta=\pi / 2,3 \pi / 2$.

Proof. The identities $\sin ^{2} \theta=(1-\cos 2 \theta) / 2$ and $\cos ^{2} \theta=(1+\cos 2 \theta) / 2$ allow us to rewrite (1.16b) and (1.17) as

$$
\begin{aligned}
\frac{d \theta}{d t} & =\frac{\kappa}{r^{2}}+\frac{(\alpha+\beta)}{2}-\frac{[(\alpha-\beta) \cos 2 \theta]}{2}, \\
H & =-\kappa \log r-\frac{(\alpha+\beta) r^{2}}{4}+\frac{\left[(\alpha-\beta) r^{2} \cos 2 \theta\right]}{4} .
\end{aligned}
$$

Using (3.1), the equation $H(r, \theta)=w$ can be rewritten as $g_{w}(r)=\cos 2 \theta$, or, by Lemma 3.3(b), as

$$
r=f_{w}(\cos 2 \theta)
$$

The latter is a simple closed curve, symmetric with respect to the $\xi$ - and $\eta$-axes, and enclosing the origin. Each trajectory of (1.16) lies on a curve (3.6) for some $w$. By (3.5),

$$
\frac{d \theta}{d t}>\frac{(\alpha+\beta)}{2}-\frac{(\alpha-\beta)}{2}>0
$$

so the motion is counterclockwise. By (3.5) and (3.6), the period $T$ is given by (3.4). By (3.4), along with Lemma 3.3(d), (e), $T$ is a decreasing function of $w$ such that $T \rightarrow 2 \pi / \sqrt{\alpha \beta}$ as $w \rightarrow-\infty$, and $T \rightarrow 0$ as $w \rightarrow \infty$. Finally, the statements about the separation of the vortices (which, by symmetry, need only be verified for $\theta$ in $[0, \pi / 2]$ ), follow from (1.16a) since the motion is counterclockwise. This completes the proof of Theorem 3.4. 
Because $f_{w}$ is a decreasing function of $w$, smaller values of $w$ correspond to larger curves; that is, if $z<w$, then the curve $r=f_{z}(\cos 2 \theta)$ encloses the curve $r=f_{w}(\cos 2 \theta)$. Thus a consequence of Theorem 3.4 is that, if two vortices are close to each other, then their period of rotation around each other is what it would be if there were no background flow, while, if the vortices are far apart, then that period is approximately what it would be if the vortices did not affect each others' motion.

3.4. The case $\alpha>\beta, \kappa<0$. The Hamiltonian $H$ defined by (1.14) has maxima at the points $\pm P$, where, in $(\xi, \eta)$ coordinates, $P=(\sqrt{-\kappa / \beta}, 0)$. Also, $H$ has saddle points at $\pm Q$, where $Q=(0, \sqrt{-\kappa / \alpha})$. The points $\pm P$ and $\pm Q$ are the only stationary points of the system (1.13). If the pair of vortices begin with relative position given by $\pm P$ or $\pm Q$, then they maintain that relative position while their center of vorticity revolves about the origin. The values of $H$ at those points are

$$
M \equiv H( \pm P)=\frac{\kappa[1-\log (-\kappa / \beta)]}{2}>\frac{\kappa[1-\log (-\kappa / \alpha)]}{2}=H( \pm Q) \equiv S,
$$

and the behavior of a trajectory lying on a level curve $H=w$ depends on where $w$ lies in relation to $M$ and $S$. As in Section 3.3, we use the function $g_{w}$ of (3.1) to explore that behavior. The following lemma gives the information we need; I omit the proof, which is similar to that of Lemma 3.3.

LEMmA 3.5. Pick $\alpha, \beta$, and $\kappa$, with $\alpha>\beta>0$ and $\kappa<0$; define $g_{w}(r), r^{*}, M$, and $S$ by (3.1), (3.2), and (3.8). Then,

(a) $g_{w}$ is decreasing on $\left(0, r^{*}\right]$ and increasing on $\left[r^{*}, \infty\right)$; $\lim _{r \rightarrow 0^{+}} g_{w}(r)=+\infty$; $\lim _{r \rightarrow \infty} g_{w}(r)>1$;

(b) $g_{w}\left(r^{*}(w)\right)$ is an increasing function of $w$ such that (i) $g_{M}\left(r^{*}(M)\right)=1$; (ii) $-1<$ $g_{w}\left(r^{*}(w)\right)<1$ if $S<w<M$; (iii) $g_{S}\left(r^{*}(S)\right)=-1$; and (iv) $g_{w}\left(r^{*}(w)\right)<-1$ if $w<S$;

(c) given $w<M$ and $u$ in $\left(g\left(r^{*}\right), 1\right]$, the equation $g_{w}(r)=u$ has exactly two solutions $r$ in $(0, \infty]$, namely, $r_{1}=f_{w}(u)$ and $r_{2}=h_{w}(u)$, where $f_{w}$ and $h_{w}$ are the inverse functions of the restrictions of $g_{w}(r)$ to the intervals $\left(0, r^{*}\right]$ and $\left[r^{*}, \infty\right)$; if $u=g\left(r^{*}\right)$ then the equation has exactly one solution, namely $f_{w}(u)=h_{w}(u)=r^{*}$;

(d) $0<f_{w}(u)<r^{*}$ and $h_{w}(u)>r^{*}$ for all $w<M$ and all $u$ in $\left(g\left(r^{*}\right), 1\right]$;

(e) for each fixed $u$ in $[-1,1], f_{w}(u)$ is an increasing function of $w$ and $h_{w}(u)$ is a decreasing function of $w$;

(f) $\lim _{w \rightarrow-\infty} f_{w}(u)=0$ and $\lim _{w \rightarrow-\infty} h_{w}(u)=\infty$.

The following definitions are helpful in describing the level curves of $H$. With $\xi$ and $\eta$ given by (1.12), and polar coordinates $r, \theta$ given by (1.7) and (1.15), we define four curves in the $\xi \eta$-plane (see Figure 3.1):

$$
\begin{aligned}
& C_{1}: r=f_{S}(\cos 2 \theta), \quad-\frac{\pi}{2}<\theta<\frac{\pi}{2} ; \quad C_{2}: r=h_{S}(\cos 2 \theta), \quad-\frac{\pi}{2}<\theta<\frac{\pi}{2} ; \\
& C_{3}: r=f_{S}(\cos 2 \theta), \quad \frac{\pi}{2}<\theta<\frac{3 \pi}{2} ; \quad C_{4}: r=h_{S}(\cos 2 \theta), \quad-\frac{\pi}{2}<\theta<\frac{3 \pi}{2} .
\end{aligned}
$$

We also define four open, connected sets: $R_{1}$ is the inside of $C_{1} \cup C_{2} \cup\{Q,-Q\}$, excluding $P$; $R_{2}$ is the inside of $C_{3} \cup C_{4} \cup\{Q,-Q\}$, excluding $-P$; $R_{3}$ is the inside 


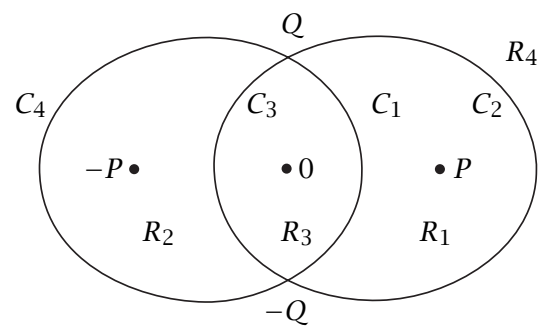

FIGURE 3.1

of $C_{1} \cup C_{3} \cup\{Q,-Q\}$, excluding the origin; and $R_{4}$ is the outside of $C_{2} \cup C_{4} \cup\{Q,-Q\}$. (The curve $C_{1} \cup C_{2} \cup\{Q,-Q\}$ encloses $P$ because $f_{S}(1)<\sqrt{-\kappa / \beta}<h_{S}(1)$ by (3.8) and Lemma 3.5(a).) Then $H\left(R_{1}\right)=H\left(R_{2}\right)=(S, M)$ and $H\left(R_{2}\right)=H\left(R_{3}\right)=(-\infty, S)$; this results from (3.8) along with (i) $\lim _{(\xi, \eta) \rightarrow \infty} H(\xi, \eta)=-\infty$, (ii) $H \equiv S$ on $\{Q,-Q\} \cup \bigcup_{i=1}^{4} C_{i}$, and (iii) $H$ has no critical points in $\bigcup_{i=1}^{4} R_{i}$.

By the Poincaré-Bendixson theorem and a corollary [2, Theorem, page 248 and Theorem 3, page 252], each region $R_{i}$ is a union of periodic orbits of (1.13). The following theorem gives more detail.

THEOREM 3.6. Fix real numbers $\alpha, \beta, \kappa_{1}$, and $\kappa_{2}$ such that $\alpha>\beta>0$ and $\kappa=$ $\kappa_{1}+\kappa_{2}<0$, define $M$ and $S$ by (3.8), let $H$ be given by (1.14), (1.15), (1.16), and (1.17), and consider a pair of vortices whose positions satisfy (1.7), (1.8), (1.9), (1.10), and (1.11). For those vortices, define $\xi$ and $\eta$ by (1.12), and put $\xi_{0}=\xi(0)$ and $\eta_{0}=\eta(0)$. Then:

(a) If $\left(\xi_{0}, \eta_{0}\right) \in R_{1} \cup R_{2}$, then the line segment joining the vortices periodically rocks from side to side in such a way that its maximum and minimum angels with the positive $\xi$-direction are $\pm \theta^{*}$, where

$$
\theta^{*}=2^{-1} \cos ^{-1} g_{w}\left(r^{*}\right)=2^{-1} \cos ^{-1}\left[(\alpha-\beta)^{-1}\left(2 \kappa e^{2 w / \kappa-1}+\alpha+\beta\right)\right]
$$

and $w=H\left(\xi_{0}, \eta_{0}\right)$. The period is

$$
T=4(\alpha-\beta)^{-1} \int_{f_{w}(1)}^{h_{w}(1)} r^{-1}\left\{1-\left[g_{w}(r)\right]^{2}\right\}^{-1 / 2} d r .
$$

The maximum and minimum length of the segment occur at the two instants in the cycle when the segment is horizontal.

(b) If $\left(\xi_{0}, \eta_{0}\right) \in \bigcup_{i=1}^{4} C_{i}$ then, as $t \rightarrow \infty$, the line segment joining the vortices tends to a vertical position. The segment's length approaches $\sqrt{-\kappa / \alpha}$.

(c) If $\left(\xi_{0}, \eta_{0}\right) \in R_{3} \cup R_{4}$ then, in $(\xi, \eta)$-coordinates, the second vortex moves around the first in a simple closed curve.

If $\left(\xi_{0}, \eta_{0}\right) \in R_{3}$, then the motion is clockwise, with period

$$
T=-8 \int_{0}^{\pi / 2}\left\{\frac{2 \kappa}{\left[f_{w}(\cos 2 \theta)\right]^{2}}+(\alpha+\beta)-(\alpha-\beta) \cos 2 \theta\right\} d \theta .
$$

The period $T$ is an increasing function of $w$ such that $T \rightarrow 0$ as $w \rightarrow-\infty$. (That is, the period is small when the vortices are close to each other.) The maximum separation $r$ of the vortices occurs when $\theta=\pi / 2,3 \pi / 2$, and the minimum when $\theta=0, \pi$. 
If $\left(\xi_{0}, \eta_{0}\right) \in R_{4}$, then the motion is counterclockwise, and the period is

$$
T=8 \int_{0}^{\pi / 2}\left\{\frac{2 \kappa}{\left[h_{w}(\cos 2 \theta)\right]^{2}}+(\alpha+\beta)-(\alpha-\beta) \cos 2 \theta\right\} d \theta .
$$

The period $T$ is an increasing function of $w$ such that $T \rightarrow 2 \pi / \sqrt{\alpha \beta}$ as $w \rightarrow-\infty$. (That is, the period is close to the background flow period when the vortices are far apart.) The maximum separation $r$ of the vortices occurs when $\theta=0, \pi$, and the minimum when $\theta=\pi / 2,3 \pi / 2$.

Proof. In proving (a), we can assume that $\left(\xi_{0}, \eta_{0}\right) \in R_{1}$; this is because (1.13) is unchanged when $\xi$ and $\eta$ are replaced by $-\xi$ and $-\eta$. Then $(\xi(t), \eta(t)) \in R_{1}$ for all $t$. The trajectory is contained in a level set $H=w$ such that $S<w<M$. As in the proof of Theorem 3.4, the equation $H=w$ can be written in the form $g_{w}(r)=\cos 2 \theta$. By Lemma 3.5(a), (b), and (c), this last equation has solutions $r$ if and only if

$$
\cos 2 \theta \geq g_{w}\left(r^{*}\right) .
$$

Since $R_{1} \subset\{-\pi / 2<\theta<\pi / 2\}$, the solutions are

$$
r=f_{w}(\cos 2 \theta), \quad r=h_{w}(\cos 2 \theta), \quad \text { where } \theta \in\left[-\theta^{*}, \theta^{*}\right] .
$$

Equations (3.15) together represent a simple closed curve; this is a consequence of Lemma 3.5(d) and the equation (from (3.10)) $f_{w}\left(\cos 2 \theta^{*}\right)=h_{w}\left(\cos 2 \theta^{*}\right)$. Therefore the motion is periodic, with the maximum and minimum values of $\theta$ stated in part (a) of Theorem 3.6. To verify the formula (3.11) for the period, we first rewrite (1.16a), for $\theta$ in $\left[0, \theta^{*}\right]$, as

$$
\frac{d r}{d t}=-2^{-1}(\alpha-\beta) r \sqrt{1-\left[g_{w}(r)\right]^{2}} .
$$

We then define $T_{f}$ and $T_{h}$ to be the amounts of time spent by the second vortex in the parts of the upper half-plane $\{\eta>0\}$ where $r<r^{*}$ and $r>r^{*}$, respectively. After separating variables in (3.16), we find that

$$
T_{f}=\frac{2}{\alpha-\beta} \int_{f_{w}(1)}^{r^{*}} \frac{d r}{r \sqrt{1-\left[g_{w}(r)\right]^{2}}}, \quad T_{h}=\frac{2}{\alpha-\beta} \int_{r^{*}}^{h_{w}(1)} \frac{d r}{r \sqrt{1-\left[g_{w}(r)\right]^{2}}},
$$

which yields (3.11). Finally, by (3.15) and Lemma 3.5(a), the smallest and largest values of $r$ are, respectively, $f_{w}(1)$ and $h_{w}(1)$; these occur when $\theta=0$. Thus the minimum and maximum separations of the vortices occur when the segment joining them is horizontal, and the proof of (a) is complete.

Part (b) is clear since the boundary of each curve $C_{i}$ is $\{Q,-Q\}$.

We prove (c) only in the case where $\left(\xi_{0}, \eta_{0}\right) \in R_{3}$; the proof for $\left(\xi_{0}, \eta_{0}\right)$ in $R_{4}$ is similar. Put $w=H\left(\xi_{0}, \eta_{0}\right)$. Then, since $w<S$, it follows from Lemma 3.5(b), (c) that the level set $H=w$ consists of two disjoint simple closed curves $r=f_{w}(\cos 2 \theta)$ and $r=h_{w}(\cos 2 \theta)$. By Lemma 3.5(d), the former is the one that lies in $R_{3}$. By Lemma 3.5(a), $d r / d \theta>0$ on the part of that curve in the first quadrant. But $d r / d t<0$ there by (1.16a), so the motion is clockwise. The statements about the vortices' separation, and 
the formula (3.12) for the period, are established as in the proofs of the corresponding facts in Theorem 3.4. The period $T$ is an increasing function of $w$ such that $T \rightarrow 0$ as $w \rightarrow-\infty$ by (3.12) and Lemma 3.5(e), (f). This completes the proof of Theorem 3.6.

Under the hypotheses of Theorem 3.6, the solutions of the linearization of (1.13) about $P=(\sqrt{-\kappa / \beta}, 0)$ have period $2 \pi / \sqrt{2 \beta(\alpha-\beta)}$. The following statements are probably true, but we have been unable to prove them: (i) if $w \in(S, M)$, then the period $T$ is a decreasing function of $w$ such that $\lim _{w \rightarrow M^{-}} T=2 \pi / \sqrt{2 \beta(\alpha-\beta)}$; (ii) $\lim _{w \rightarrow S} T=\infty$.

ACKNowledgement. The author is grateful to Franklin and Marshall College for financial support of this project.

\section{REFERENCES}

[1] A. J. Chorin and J. E. Marsden, A Mathematical Introduction to Fluid Mechanics, 3rd ed., Texts in Applied Mathematics, vol. 4, Springer-Verlag, New York, 1993. MR 94c:76002. Zbl 0774.76001.

[2] M. W. Hirsch and S. Smale, Differential Equations, Dynamical Systems, and Linear Algebra, Pure and Applied Mathematics, vol. 60, Academic Press, New York, 1974. MR 58\#6484. Zbl 0309.34001.

[3] Y. Kimura and H. Hasimoto, Motion of two identical point vortices in a simple shear flow, J. Phys. Soc. Japan 54 (1985), no. 11, 4069-4072. MR 87b:76034.

[4] H. J. Lugt, Introduction to Vortex Theory, Vortex Flow Press, Incorporated, Potomac, Maryland, 1996. MR 2001e:76001.

[5] C. Marchioro and M. Pulvirenti, Mathematical Theory of Incompressible Nonviscous Fluids, Applied Mathematical Sciences, vol. 96, Springer-Verlag, New York, 1994. MR 94k:76001. Zbl 0789.76002.

[6] I. Percival and D. Richards, Introduction to Dynamics, Cambridge University Press, Cambridge, 1982. MR 84e:70001. Zbl 0499.70027.

Robert M. Gethner: MAthematics Department, Franklin and MARshall College, LANCASTER, PA 17604, USA

E-mail address: r_gethner@fandm.edu 


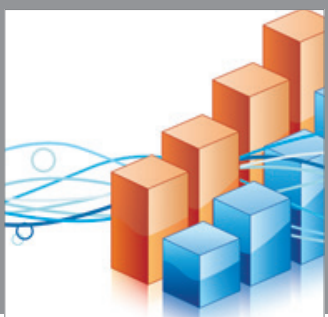

Advances in

Operations Research

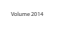

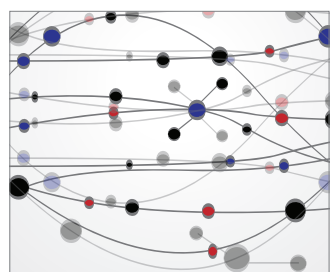

\section{The Scientific} World Journal
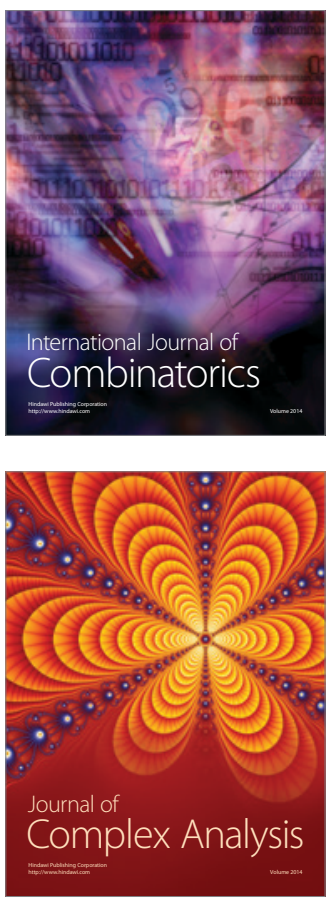

International Journal of

Mathematics and

Mathematical

Sciences
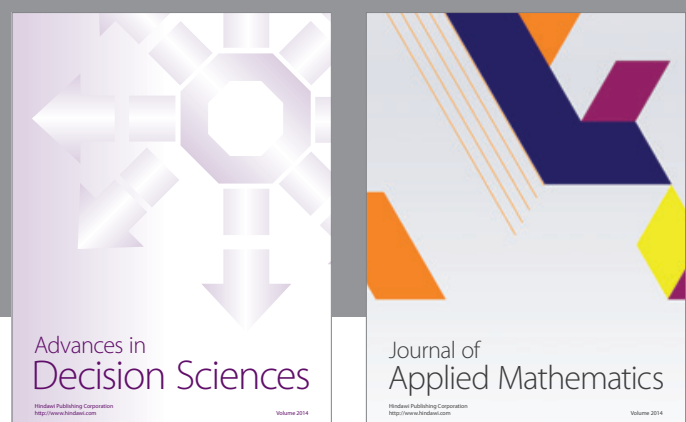

Journal of

Applied Mathematics
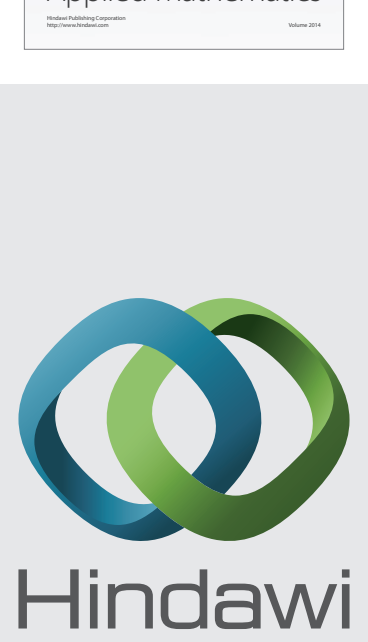

Submit your manuscripts at http://www.hindawi.com
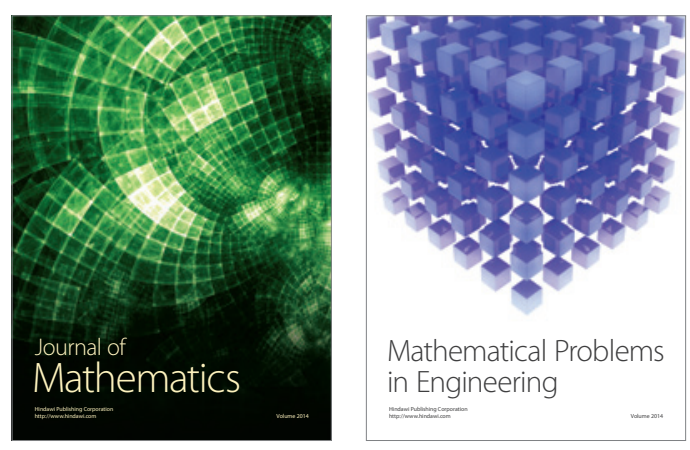

Mathematical Problems in Engineering
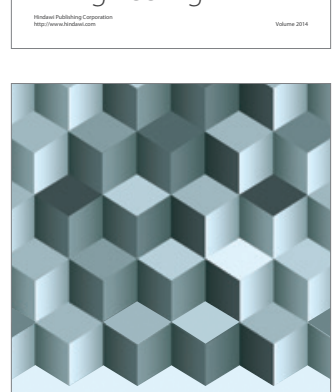

Journal of

Function Spaces
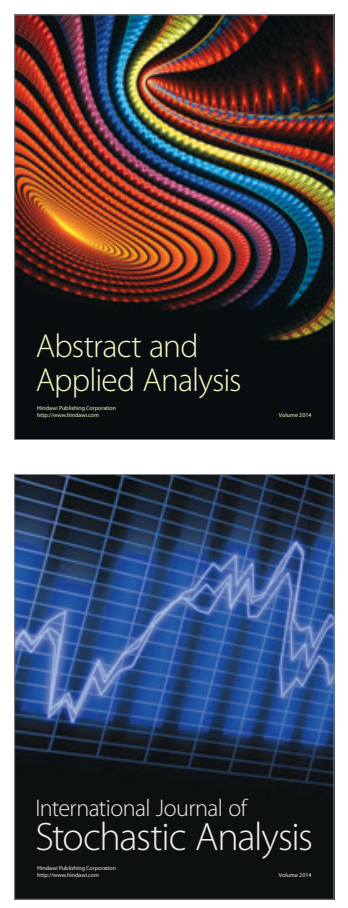

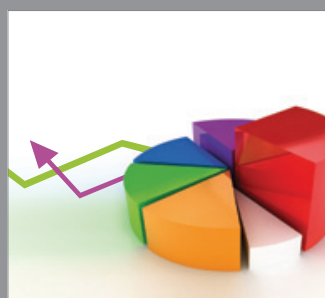

ournal of

Probability and Statistics

Promensencen
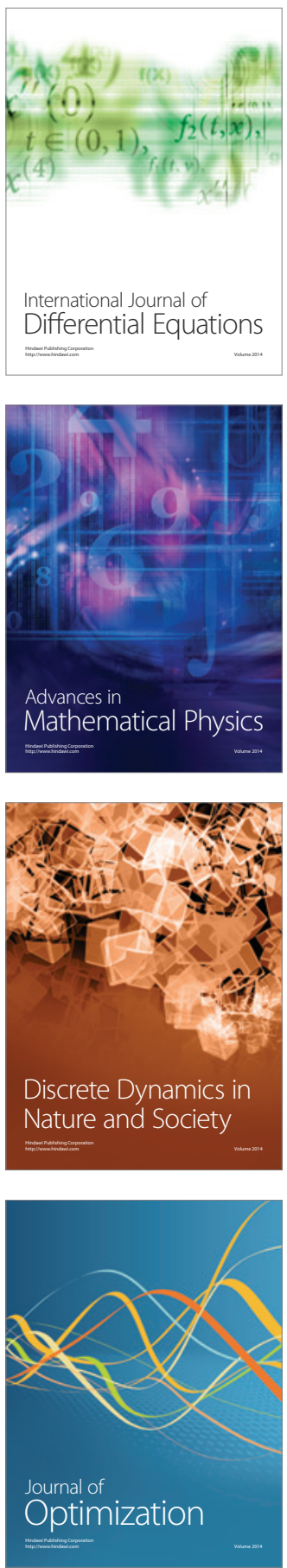